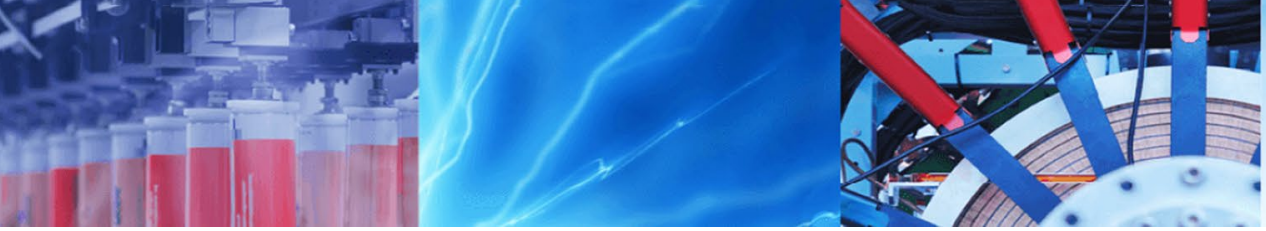

Research Article

\title{
Imaging, deposition, and self-assembly of CTAB stabilized gold nanostructures
}

\author{
Imtiaz Ahmad' ${ }^{1}$ Rahim $\operatorname{Jan}^{2} \cdot$ Hidayat Ullah Khan ${ }^{1} \cdot$ Akhlaq Hussain $^{1} \cdot$ Sajjad Ahmad Khan ${ }^{3}$
}

Received: 23 July 2019 / Accepted: 4 February 2020 / Published online: 20 May 2020

(c) Springer Nature Switzerland AG 2020

\begin{abstract}
True picture of the nanoparticle arrangements is significant owing to their unique applications that are generally linked with their collective response within the self-assembled arrays over the underlying surface. Surfactant such as cetyltrimethylammonium bromide (CTAB) stabilized gold nanostructures on two different surfaces such as silicon dioxide and highly oriented pyrolytic graphite $\left(\mathrm{SiO}_{2}, \mathrm{HOPG}\right)$ have been investigated via using well known and widely used imaging techniques. These frequently employed substrates for the study of nanoparticles self-assembly have been used to study their images after complete evaporation of the suspension droplet. The resulting images of nanoparticle deposits via various techniques revealed contrasting topographies; some of the self-assembled features over a particular surface are highlighted in one technique whereas hidden in other technique. As CTAB molecules are not merely use to stabilizing the gold core during synthesis but these molecules also for all intents and purposes exist in suspension with the gold nanostructures. Therefore, for such multi-nano systems, evaluation of nanoparticles images by a single imaging technique is generally misleading. This work will highlight the contrasting results acquired using various imaging techniques such as high resolution scanning electron microscope, helium ion microscope, atomic force microscope, and scanning tunneling microscope.
\end{abstract}

Keywords Self-assembly $\cdot \mathrm{CTAB} \cdot$ Gold nanorods $\cdot \mathrm{HOPG} \cdot \mathrm{SiO}_{2}$

\section{Introduction}

Gold nanorods are attracting a great deal of attention due to their imminent applications in numerous fields. Owing to their strong optical response, ordered arrays of plasmonic nanostructures are promising candidates for the novel optical sensors, solar cells, and many other applications [1-6]. In this respect, control over the assembled area, overall deposited structures, and homogeneity within the ordered arrays are essential. Ultimate control over self-assembly of nanorods from few $100 \mathrm{~nm}$ to few microns range and at specific locations over the surface still requires further research $[7,8]$.
Surfactant molecules such as cetyltrimethylammonium bromide (CTAB) is a prime candidate for the synthesis of high yield and nearly monodispersed gold nanorods [9-11]. Also, these molecules in suspension if present in excess will facilitate shape-separation effect and act like depletants. However, from the medical point of view CTAB molecule is toxic in nature and hence has poor biocompatibility profile [12]. Such stabilizing adhesive molecules, micelles, polymeric composites, surfactants and linkers are considered in wide range of studies due to their versatile uses and applicability in various fields [13-18]. CTAB in suspension with nanoparticles also helps to direct self-assembly and shape-separation process as well as modify surface

\footnotetext{
$\triangle$ Imtiaz Ahmad, imtiaz@uop.edu.pk| 'Department of Physics, University of Peshawar, Peshawar 25120, KP, Pakistan. ${ }^{2}$ School of Chemical and Materials Engineering, National University of Sciences and Technology, Islamabad 44000, Pakistan. ${ }^{3}$ Department of Statistics, Islamia College Peshawar, Peshawar, Pakistan.
} 
wettability $[19,20]$. During the synthesis of gold nanorods, the adsorbed surfactant is supposed to form a bilayer on the surface of the nanorods [21] whereas bromide ions form a complex with other reactants in the suspension. This process changes the absorption of the CTAB on the gold surface and therefore changes the growth mechanism [22]. To reduce CTAB amount in suspension these solution are centrifuged around 15,000 rpm for $10 \mathrm{~min}$ [23-25]. This process is generally known as purification of suspension and besides CTAB it removes other excess chemicals from solution. However, numerous techniques have been employed to partially remove or exchange the CTAB surfactant, purification of CTAB and replacing with surface active materials like thiols (PEGylated) and other polymers $[26,27]$. However, during the surfactant exchange process, CTAB-coated gold nano structures are always observed disrupted and consequently leads to aggregation of nanoparticles. Moreover, excess CTAB molecules is often observed in suspension after the surfacemodification techniques [28].

The scanning helium ion microscope (SHIM) bear a close resemblance to a scanning electron microscope (SEM) in its overall architecture with few minor adjustments. The ion source provides a beam of focused, aperture, and scanned helium ions over the substrate. The angle of convergence is typically smaller (five times) than a SEM, hence the field depth is five times greater for the similar conditions. The scanning helium ion microscope images can be constructed from a variety of signals from several detector options. Most common signal to evaluate features on the substrate surface is form by the secondary electrons. Secondary electrons in the scanning helium microscopy produces almost entirely from the primary beam, therefore it generally holds detailed information about the surface chemistry. Some of the intriguing results of scanning helium ion microscope (SHIM) are recently presented elsewhere [29-32].

These days metallic nano-particles can be synthesized in variety of shapes [33-36] and sizes [37, 38], allowing diverse applications. In general, to uncover the complete potential of these nanostructure based applications [39, 40], it is important to realize and characterize the wide range of fascinating properties of these nano-entities. In their native form, high resolution imaging of these metallic nanoparticles provide important compositional and structural information. It is therefore essential to understand not only the shape but the presence of surfactant molecules also effects the properties associated with the ordered arrays of nano-particles [41].

Imaging techniques are useful to study assembly of micro/nano superstructures on various surfaces and scanning areas. However, some of the imaging techniques are good in one way while deceptive in other. Some are having advantage of large scan area while other had limitation of small scan area. Similarly, some imaging techniques hide some features while other highlights them within the deposits. Importantly, if someone is interested to see pure gold nanoparticle assembly, they should use SEM for imaging. However, if someone want to see the whole scenario of the deposits and to understand their detail insight, it is essential to use some additional imagining techniques. Also, in case of multi-component (nanoparticles plus surfactant molecules) deposits over the substrate, it is important to investigate even a tiny details on the surface for the reason that the presence of unwanted CTAB molecular arrays will not only disrupt the imaging but also hinder the plausible application associated from the unadulterated gold nanorod assembly. These assembled superstructures are relevant in the areas of electronics $[42,43]$, biomedical studies [44-46], sensing [47-49], photonics [50-53], and SERS [54-56] applications. Therefore, to evaluate nanoparticle superstructures and their revealing true pictures on various surfaces is a very crucial for future research.

For multi-component system like gold nanorods and CTAB molecules, it has been observed that CTAB molecules not only stabilizes the gold core but also prevent them from agglomeration during synthesis, self-assembles in the form of stripes on HOPG surface [57-59]. Also, as Highly Ordered Pyrolytic Graphite (HOPG) surface is nonwetting in nature $[60,61]$ whilst the CTAB surfactant molecules have a wetting end group and a nonwetting tail [62]. Hence, to prevent their nonwetting tail from water, these surfactant molecules assemble in the form of spherical and cylindrical micelles in suspension $[62,63]$. Such attractive self-assembling of CTAB molecules both in suspension as well as on the surface requires detail investigation using state of the art imaging techniques.

In this paper, our aim is to use some of the well-known imaging techniques like SEM, HIM, AFM, and STM to study ordered arrays of gold nanorods on $\mathrm{SiO}_{2}$ and HOPG surfaces. Besides interesting convictive self-assembly of nanorods, the main aim behind this work is to highlight the similarities and differences in the imaging techniques for such multi-component system. Therefore to evaluate the contrasting behaviour of HIM and SEM, first it was important to locate a particular location on the surface for both the cases and then compare them with each other. $\mathrm{HIM}$ and SEM imaging for that reason is performed on $\mathrm{SiO}_{2}$ surface. However, AFM and STM are employed to image the gold nanoparticle deposits on the HOPG. This work will help to understand better the applications desired from the ordered arrays of gold nanorods owing to the fact that the presence of CTAB arrays are usually hidden in the SEM images. Existence of CTAB molecules everywhere around the gold nanorods may impede the incident and reflected signals from the ensemble arrays. Consequently, many 
applications that are associated with the gold ordered arrays will also be affected.

\section{Experimental}

\subsection{Materials}

Hydrogen tetrachloroaurate $\left(\mathrm{HAuCl}_{4} \cdot 3 \mathrm{H}_{2} \mathrm{O}, 99.999 \%\right.$, Aldrich), sodium borohydrate $\left(\mathrm{NaBH}_{4}, 99 \%\right.$, Aldrich), ascorbic acid (AA, 99\%, Merck), hydrochloric acid ( $\mathrm{HCl}, 37 \%$, Merck), cetyltrimethylammonium bromide (CTAB, Aldrich, $98 \%)$, silver nitrate $\left(\mathrm{AgNO}_{3}, 99 \%\right.$, Acros), were used. For synthesis, all water was of Milli-Q quality $(18.2 \mathrm{Mcm})$, obtained in a Simplicity 185 system (Millipore).

\subsection{Synthesis}

2-step seed mediated method explained by Nikoobakht and El-Sayed [64] to make the gold nanostructures used for this study. First of all, CTAB coated seed particles were prepared by mixing $25 \mu \mathrm{l}$ of $\mathrm{HAuCl}_{4}(0.1 \mathrm{M})$ in $10 \mathrm{ml}$ of CTAB (0.1 M). After that $60 \mu$ l of ice cold $\mathrm{NaBH}_{4}(0.1 \mathrm{M})$ was added with constant stirring for $5 \mathrm{~min}$. The solution swiftly turns light brown, indicative of the creation of gold seeds. The resulting solution was set aside at room temperature for $2 \mathrm{~h}$.

In second step, for the synthesis of gold nanorods, the growth solution was made by mixing $50 \mu \mathrm{l}$ of $\mathrm{HAuCl}_{4}$ $(0.1 \mathrm{M})$ with $10 \mathrm{ml}$ of CTAB $(0.1 \mathrm{M})$. This solution was kept at $30{ }^{\circ} \mathrm{C}$ for 25 minutes to dissolve the CTAB completely with rigorous stirring. Hereafter, $20 \mu \mathrm{l}$ of $\mathrm{AgNO}_{3}(0.1 \mathrm{M})$ was added at room temperature. Then $70 \mu$ of ascorbic acid $(0.1 \mathrm{M})$ was introduced, and followed by $100 \mu \mathrm{l}$ of $\mathrm{HCl}$ (1 M). Lastly, $25 \mu \mathrm{l}$ of seed solution was introduced into the growth solution, and was left undisturbed for overnight at room temperature.

Before use, prepared solution was centrifuged at 15,000 rpm for 10 min to remove the excess CTAB; this translates into $21,130 \mathrm{~g}$ using the geometry of the rotor (radius $8.4 \mathrm{~cm}$ ). These suspensions were stored in the refrigerator.

\subsection{Deposition}

Both $\mathrm{SiO}_{2}$ and $\mathrm{HOPG}$ substrates were ultrasonically cleaned in acetone for $10 \mathrm{~min}$ at room temperature. Then the substrates were rinsed using ethanol and distilled water and followed by drying it in a nitrogen flow. $2 \mu$ droplets of the nanorod suspensions were placed on the clean substrates and subsequently allowed to evaporate at room temperature. Within 2-3 $\mathrm{h}$ the solvent was completely evaporated. HOPG was used for AFM and STM analysis, to evaluate the presence and deposition of excess CTAB molecules around the gold nanorods.

\subsection{Characterization}

Measurements on HIM were carried out using an ultra-high vacuum (UHV) Orion Plus helium ion microscope from Zeiss. The microscope is built-in with an Everhardt-Thornley (ET) detector for the detection of Secondary Electron (SE). A micro-channel plate positioned below the last lens and just above the sample permits the qualitative analysis of Backscattered Helium (BSHe). This detector provides images, where dark region corresponds to light elements, with low backscatter yield, and bright regions, having a high backscatter probability, which correspond to heavy elements in the sample.

High-resolution scanning electron microscopy (Merlin Field Emission SEM (FE-SEM) Zeiss 1550 system) was used for imaging of our samples with nanoparticle deposits; typical voltages in the range $0.1-30 \mathrm{kV}$ are accessible. This microscope is fitted with a on-axis in-lens as well as a high efficiency off-axis secondary electron detectors. In this study, in-lens detector has been used, which is a high efficiency detector for both SE1 and SE2 and gives excellent imaging to the location in the beam path and the grouping with the electrostatic/electromagnetic lens. This particular detector is superb at low voltages for small working distances.

AFM imaging was carried out in tapping mode using Agilent 5100 Atomic Force Microscope using HQ:NSC35/Al probes (Mikromasch) with a spring constant of $5-16 \mathrm{~N} / \mathrm{m}$ and a resonance frequency of $150-300 \mathrm{kHz}$.

STM images are taken using NaioSTM (Nanosurf, version 3.1) for the analysis of the CTAB coated $\left(\mathrm{C}_{19} \mathrm{H}_{42} \mathrm{BrN}\right)$ gold nanorods. The set points for the tip-current and tip-voltage are $1 \mathrm{nA}$ and $1.5 \mathrm{~V}$, respectively, to see the nanoentities on the HOPG.

\section{Results}

\subsection{Deposits on $\mathrm{SiO}_{2}$ surface}

Suspension comprises of gold nanorods and CTAB molecules deposit nanoentities in a coffee-stain like ring after evaporation of the solvent. A portion of a coffee-stain ring is shown in Fig. 1a, where smooth clean area at the extreme left is the outer region of the coffee-stain ring on the $\mathrm{SiO}_{2}$ surface whereas unclean relatively dark interior region after the dense coffee-stain ring comprise of mostly CTAB deposits. Identical regions at the outer edge of the coffee-stain ring are characterized with the help of HIM and SEM. In Fig. 1b, HIM image of nano-deposit displays 

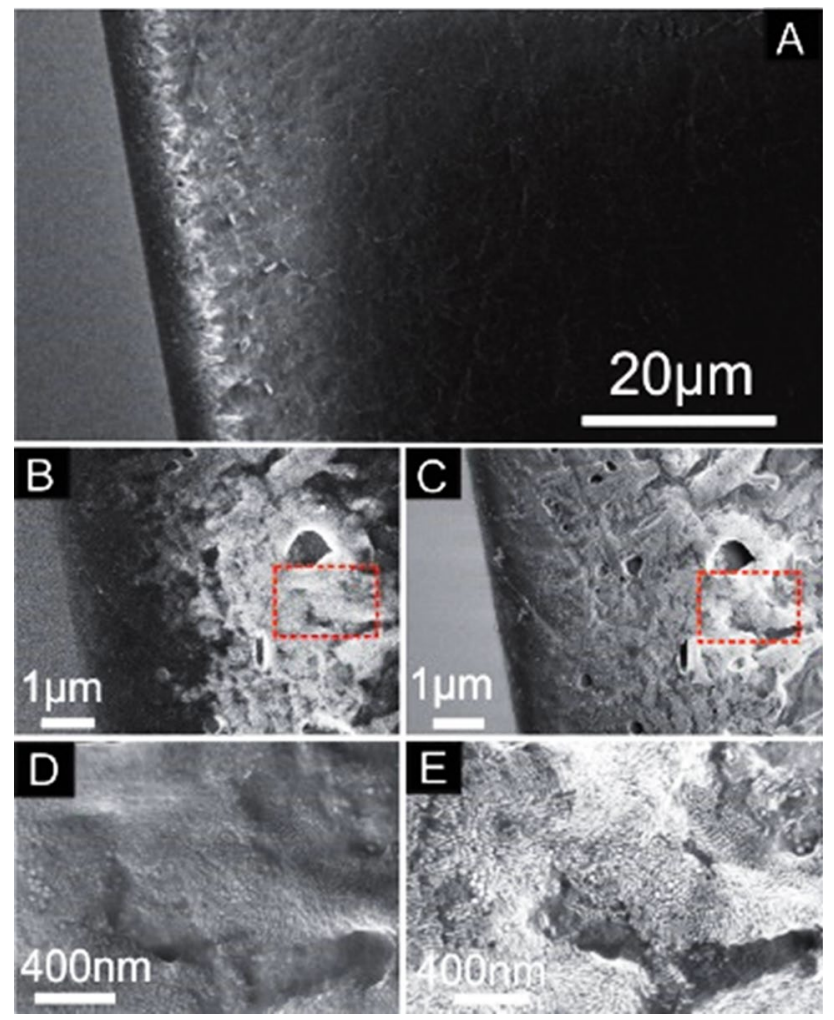

Fig. 1 a SEM image of the gold nanorods deposit in a portion of coffee-stain ring. b, c HIM and SEM image of the same area within the coffee-stain ring respectively. $\mathbf{d}$, e HIM and SEM images of the red dashed regions in $\mathbf{b}, \mathbf{c}$ respectively

some dark and bright regions, where dark regions display very few nanorods and bright regions comprises of selfassemble gold nanorods. On the other hand, Fig. 1c shows SEM image of the identical region of self-assembled gold nanorods with almost no CTAB coverage in the vicinity of these deposits. Also, a detail analysis is shown in Fig. 1d, e which are essentially the enlarge images of red dashed rectangular regions in Fig. 1b, c, where Fig. 1d is the HIM image which reveal blur picture of the gold nanorod assembly, however, Fig. 1e is the SEM image that evidently shows self-assembly of gold nanorods at the identical regions with no blurring effect at all.

To analyze the situation with a lesser CTAB contents in suspension with gold nanorods, similar suspensions of gold nanorods are placed in a refrigerator for overnight. It had been observed during our experiments that keeping suspension in refrigerator $\left(0-4{ }^{\circ} \mathrm{C}\right)$ for nearly $24 \mathrm{~h}$ results nanoparticles presence in the supernatant with less $C T A B$ contents. At that temperature, generally CTAB molecules in the gold nanorod suspension settles down in the form precipitate and can be seen inside the glass vessel even by an unaided eye. Therefore, in such suspensions the upper solution contain significantly less amount of CTAB and
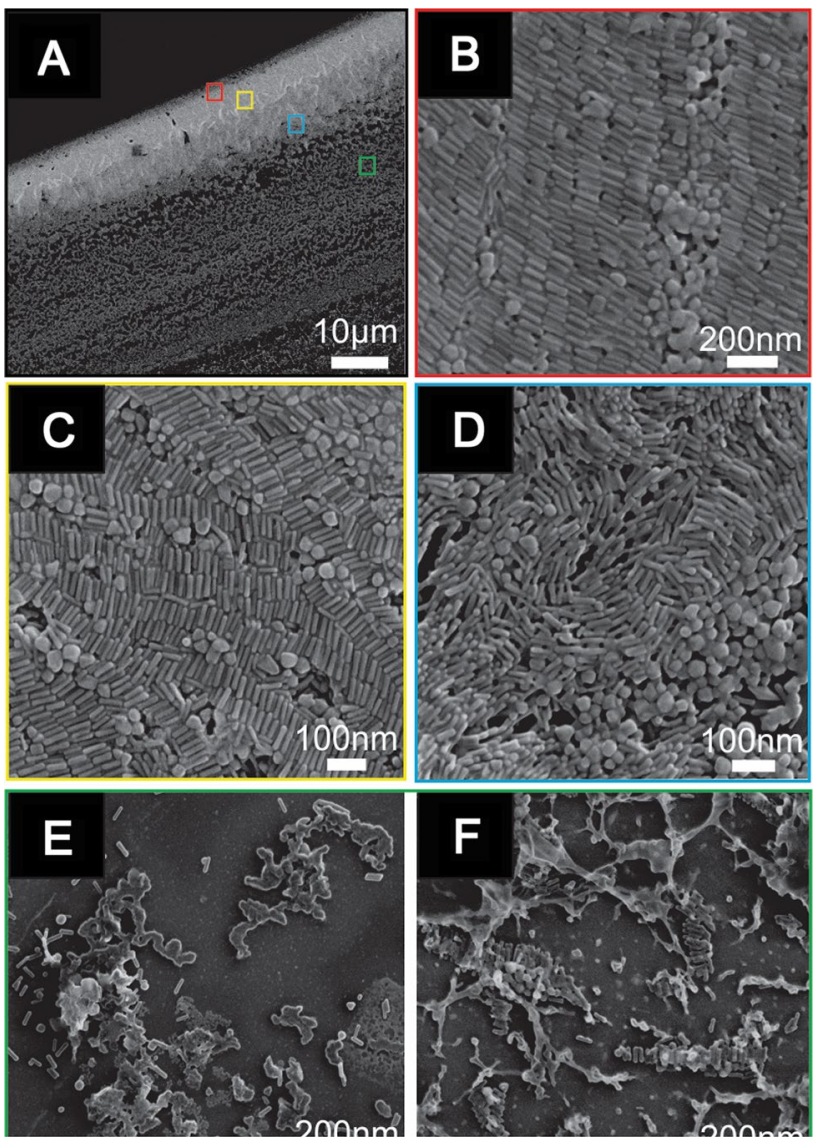

Fig. 2 HIM images of a nanoparticle deposits form a coffee-stain ring, b-d deposits of gold nanoparticles from inner to central region of the coffee-stain ring, e, fCTAB and nanoparticles at the inner regions of coffee-stain ring

consequently a droplet of such nano suspension can be used for the deposition. Nanoparticles within such deposits after evaporation of the aqueous solution are then characterized by using HIM and SEM.

Results of HIM images at various positions (color regions in Fig. 2a) of the coffee-stain ring shows nearly no blurring around the gold nanoparticle deposits as shown in Fig. 2a-f. Red, yellow, blue, and green rectangular regions in Fig. 2a are enlarged in Fig. 2b-e respectively. Assembly of side-by-side arranged gold nanorods displaying order-to-disorder transition within the coffee-stain ring like deposits, as one moves away from the outer-edge to inner-edge of the coffee-stain ring as depicted in Fig. 2b-f. Images in Fig. 2 are showing no sign of blurring or coverage of CTAB except in Fig. 1b, f, where nanoparticles are blurry and seems like connected with some gel-like substance. However, these gel-like deposits are away from the densely assembled nanorods within the coffee-stain ring (blue and green rectangles in Fig. 2a).

Similarly SEM images at the various positions (color rectangular regions in Fig. 3a) of the coffee-stain ring

\section{SN Applied Sciences}


Fig. 3 SEM images of a nanoparticles form coffee-stain like ring, $\mathbf{b}$-d deposits of nanoparticles from outer (red rectangle in a to central region (yellow rectangle in a) of the coffeestain ring, $\mathbf{e}, \mathbf{f}$ inner regions of coffee-stain ring as shown by the blue and green rectangles in a
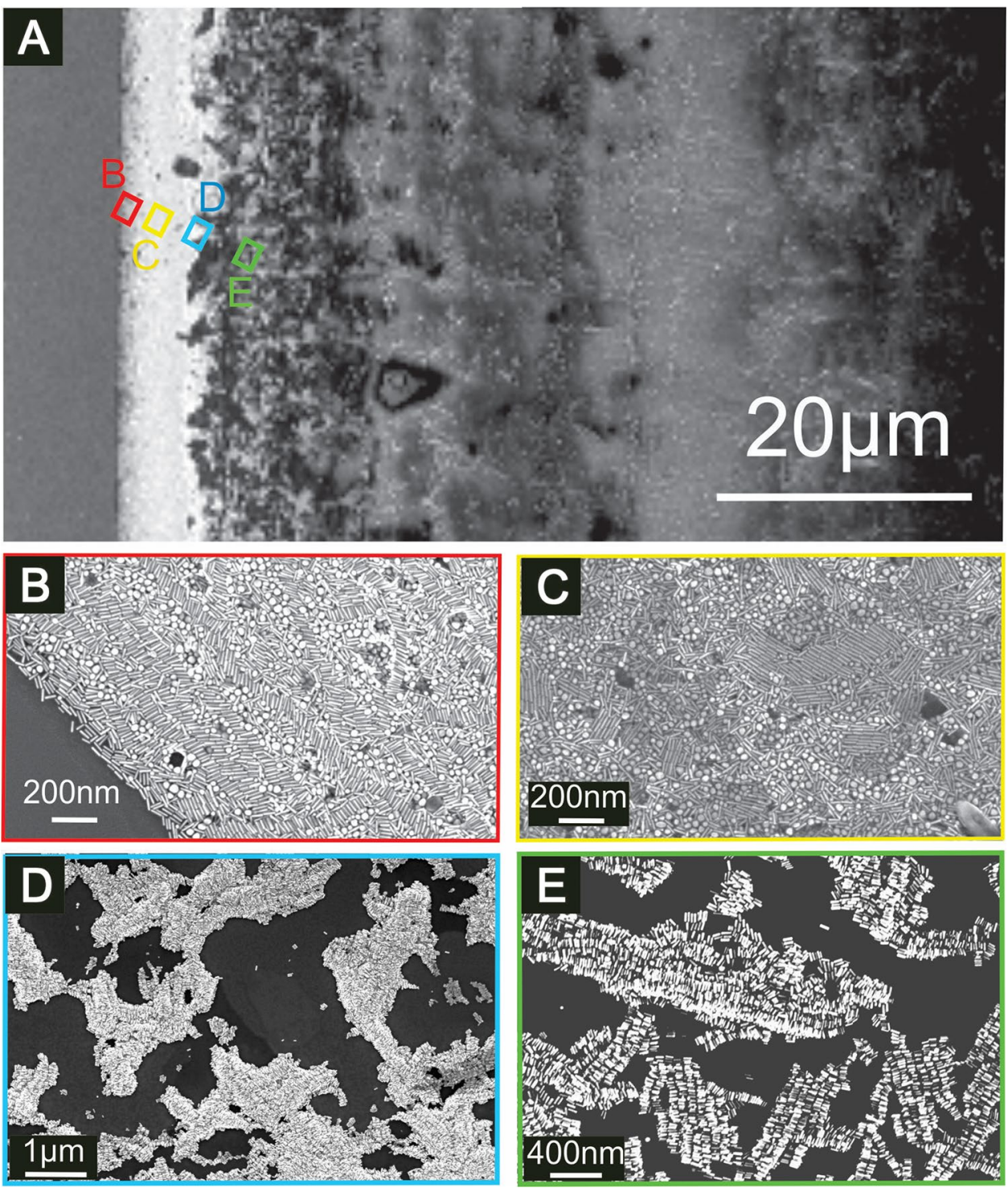

are shown in Fig. 3. The colorful rectangular areas at various locations of the deposited nanoparticles in Fig. 3a are displayed enlarged in Fig. 3b-e respectively. At the outer-edge of the coffee-stain ring, nicely assembled arrays of nanorods and some spheres can clearly be seen without any blurring effect or presence of CTAB. All SEM images are showing exclusively nanoparticles nicely self-assembled at various positions of the coffeestain like ring. Noticeably, it seems that there exist nothing in between nanorods that causes blurring within the nanoparticle deposits. However, after bright regions of such deposits (see Fig. 3a) of nanoparticles within the ring like stain, slight bright areas displays more $C T A B$ deposits than nanoparticles.

\subsection{Deposits on HOPG surface}

To further analyze CTAB stabilized gold nanorod suspensions, a droplet of such suspension was dried on HOPG to study using the AFM. Interestingly, AFM images revealed that gold nanorods self-assembles over the places on HOPG where CTAB molecules are already self-assembled and forming a carpet-like situation for the gold nanorods to settle on it. Relatively brighter regions in Fig. 4a-d are showing gold nanorods whereas slightly bright and relatively dark regions are signifying the presence of $C T A B$ deposits on HOPG surface. Furthermore, AFM images in Fig. $4 \mathrm{e}$, $\mathrm{f}$ clearly shows nanorods under the self-assembled arrays of CTAB molecules. To further illuminate the buried 
Fig. 4 a-d AFM images of nanoparticles deposited on top of CTAB ensembles on terraces and step-edges of HOPG. $(E, F)$ encircled regions shows nanorods underneath $C T A B$ stripes

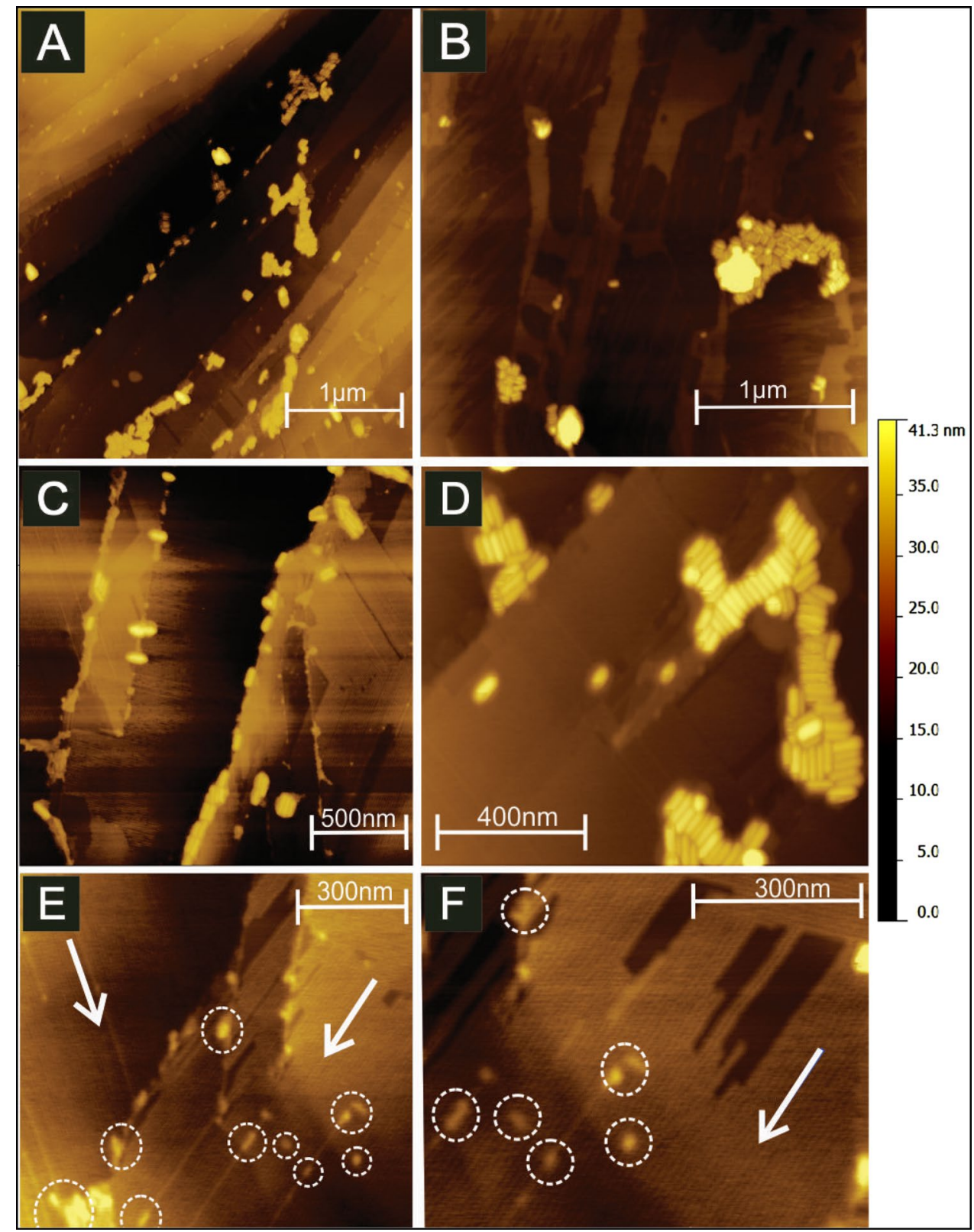

nanorods, some of these regions are displayed within the dashed white 9 circular sections whereas white arrows in Fig. $4 \mathrm{e}, \mathrm{f}$ are demonstrating the direction of the selfaligned CTAB molecules. Also, close examination of Fig. $4 d$ shows large islands of gold nanorod deposits settled on top of the CTAB layers.

For STM analysis, owing to small scanning area $(50 \mathrm{~nm} \times 50 \mathrm{~nm})$, it was very difficult to locate gold nanorods under the STM tip. However, after extended searching on the surface at various locations on the sample, eventually it become possible to locate some of the nanorod deposits. At some sites on the HOPG, self-assembled arrays of gold nanorods are observed, covered under the thick CTAB layers as shown in Fig. 5a, b. STM images of gold nanorods are shown in Fig. 5c, d, where Fig. 5c particularly display an isolated gold nanorod and also elucidate a trace of CTAB around it. Likewise, within the same figure (Fig. $5 \mathrm{C}$ ) color points on the HOPG surface are shown by the circles of different colors (green $=C T A B$, red $=$ gold nanorod, blue $=\mathrm{HOPG}$ ) highlighted by the dashed arrows. For colored points in Fig. $5 c, 1-V$ curves are plotted in Fig. 5e, where green curve shows I-V for CTAB deposits, blue curve is the plot for HOPG, and red curve display the I-V behavior of CTAB coated gold nanorod. Confirming the general trend of current and voltage behaviour that one can expect from CTAB, HOPG, and gold nanorod. 

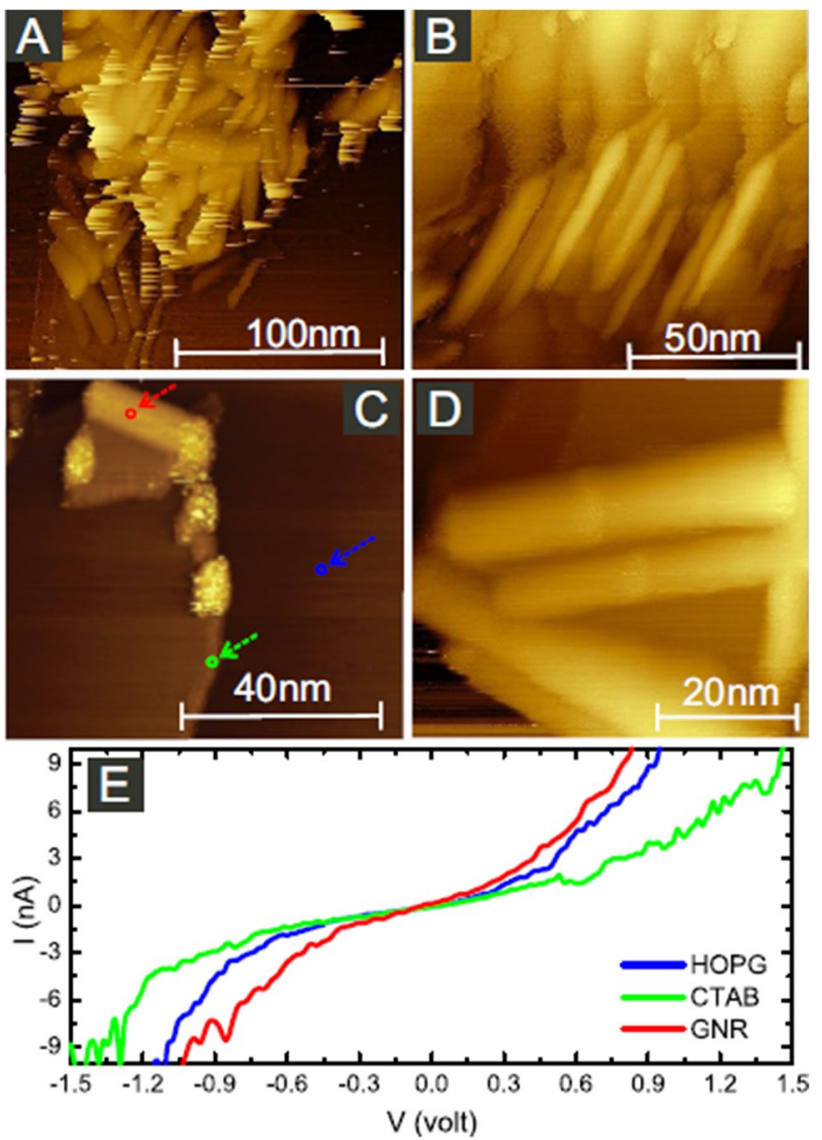

Fig. 5 Images on HOPG; a, b STM images of gold nanorods under thick layers of CTAB, $\mathbf{c}$, $\mathbf{d}$ isolated gold nanorods with traces of CTAB while $\mathbf{d}$ showing tip artifact by showing double images of single nanorod, $\mathbf{f}$ IV-plots of the points in $\mathbf{c}$ represented by dotted red, blue, and green arrows are plotted

\section{Discussions}

Two droplets of gold nanorod suspension deposited over two different surfaces ( $\left.\mathrm{SiO}_{2}, \mathrm{HOPG}\right)$ are allowed to evaporate at room temperature. Deposits of nanoparticle arrays within the coffee-stain like ring are characterized by using SEM and $\mathrm{HIM}$ for $\mathrm{SiO}_{2}$ surface whereas on HOPG substrate AFM and STM are employed to further evaluate CTAB deposits all over the surface in vicinity of gold nanorods self-assembly. Summary of the multi-nanoentities deposited over the substrates are précised schematically in Fig. 6.

Imaging a system like CTAB stabilized gold nanorods using various techniques showed some hidden features that are generally not realized if one only uses well known and widely used imaging technique such as SEM. On the basis for presented results, it can easily be realized that it is not only gold core that can be visualized on the surface but there exist CTAB molecules that are always present in suspension within the droplet and on the surface together with the deposits. Such molecules are deposit underneath as well as above the gold nanorods self-assembled arrays. Moreover, beside their presence allover surface, gold crystals are also stabilized by the double layers of CTAB molecules to stop further growing during synthesis [65].

It has been observed that SEM images show more often gold nanorods than the surrounding molecules of CTAB. As evident at every position within the coffeestain ring, SEM merely visualized gold nanorod selfassembled arrays. However, away from the coffee-stain ring, deposits are typically comprised of CTAB junks owing to their light weight sweeps away towards the inner region of the terminating droplet. Consequently, relying solely on SEM imaging do not show the true picture of the deposited nanoentities. Hence, on the basis of such results relevant uses that are expected from gold self-assembled arrays. However, SEM does show more
Fig. 6 Scheme of showing evaporation that leaves behind coffee-stain like deposits. HIM and SEM images are taken at the identical location at the outer edge of the coffee-stain ring on $\mathrm{SiO}_{2}$ surface while AFM and STM shows the existence of CTAB on the HOPG surfaces within the deposits

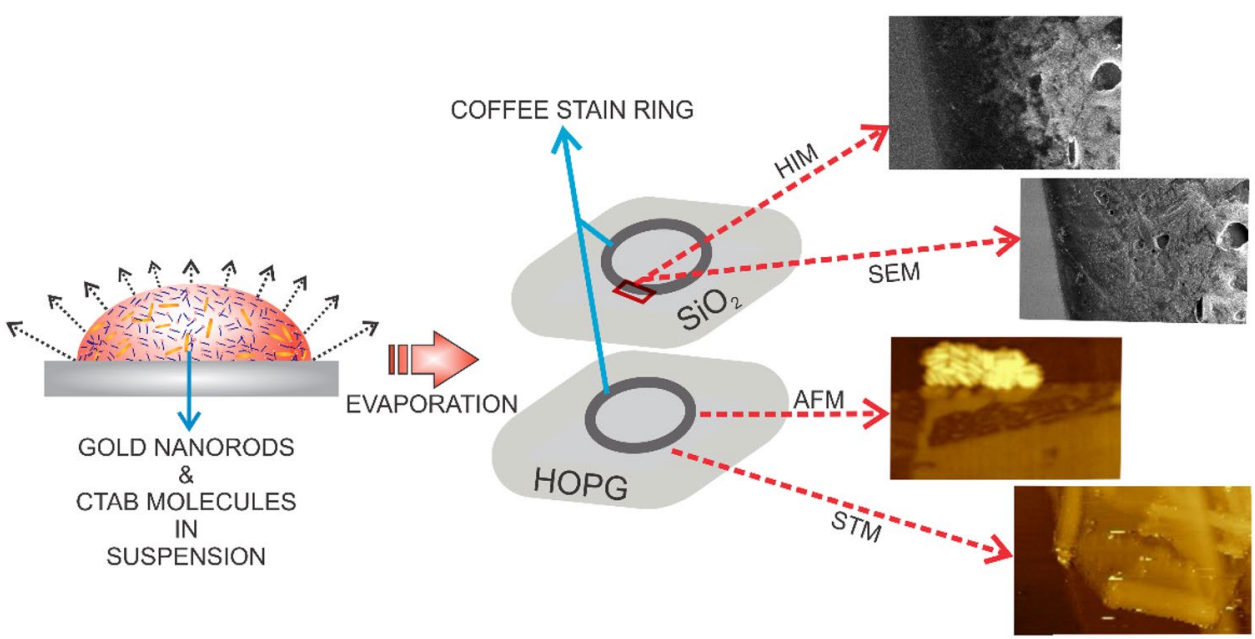

SN Applied Sciences A SPRINGER NATURE journat 
effectively the assembled arrays of gold nanorods without much difficulties.

As for as HIM imaging is concern, similar regions on $\mathrm{SiO}_{2}$ substrate showing gold nanorods much clearly in SEM than in the HIM. Imaging by HIM showing that gold nanorods are not alone rather these nanoparticles are buried in some places under massive layers of CTAB while in other places these nanoparticles were observed under the thin layer of CTAB molecules. Such results will help those researchers who merely rely merely on SEM results. Such vast presence of CTAB will result interruption in the possible applications associated with selfassembled arrays of gold nanorods. Hence information about their factual presence with the deposits must be monitored clearly. Evidently, HIM excels to show more CTAB than the gold nanorods on the surface.

To study more details of deposited nanoentities, it was required to dry similar suspension droplets on the HOPG. As HOPG is conducting nature, this allow us to study these deposits under the AFM and STM tip. AFM images showed very interesting images of nano suspensions on the HOPG surfaces. Self-assembled gold nanorods are observed on the surface surrounded by the CTAB self-assembled arrays everywhere on the HOPG. These CTAB molecules are observed underneath as well as on top of the gold nanorod deposits. Moreover, it was also observed that these CTAB molecules self-assembled on the surface of the HOPG. Such assemblies of CTAB molecules are reported in detail elsewhere $[56,66]$.

STM analysis is performed aiming to observe two different possibilities; regions populated with gold nanorods and regions comprising of isolated gold nanorods. Owing to limited scan length, it was not that easy to locate these possibilities during STM imaging. There the situation largely similar to the one we observed in the AFM imaging. Traces of CTAB molecules are observed around the gold nanorods. Also, I-V characterization of deposited entities on the HOPG surface
Fig. 7 a Size of a coffee-stain ring (in Fig. 1a) amounts to $6 \mu \mathrm{m}$. b Line scan of HIM and SEM images in Fig. 1b, $c$
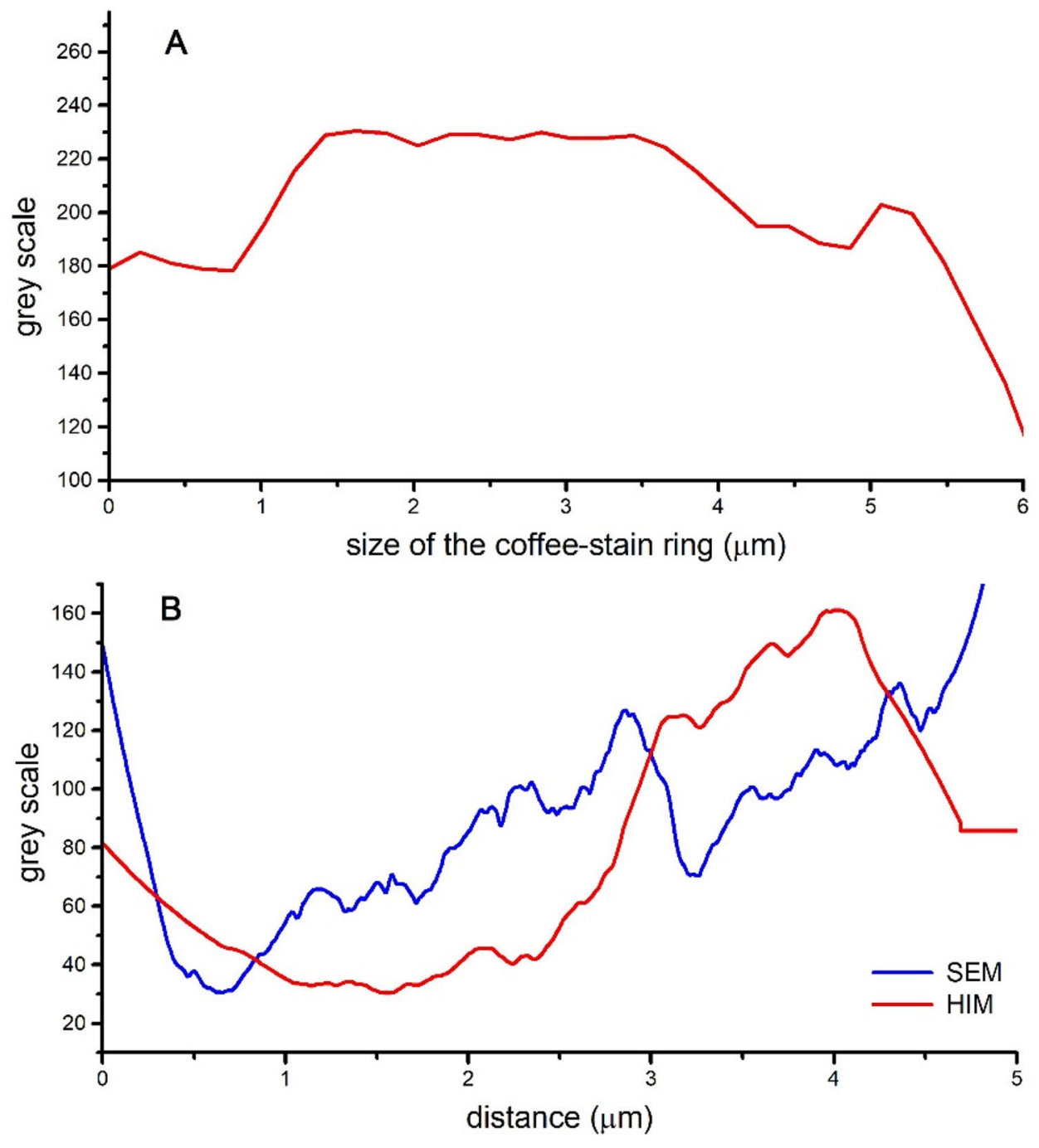

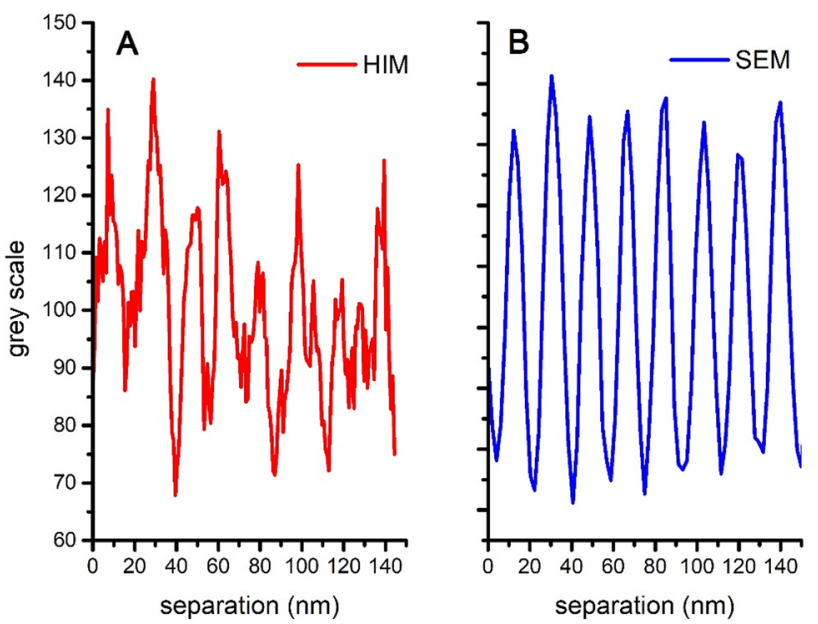

Fig. 8 a, b Line scans of side-by-side assembled gold nanorods of HIM and SEM images

showed that CTAB stabilized gold nanorod is more conducting than the HOPG and CTAB. Consequently, identifying each individual component of the system.

Quantitatively analysis of HIM and SEM images clarifies further that HIM images do not able to screened surfactant molecules efficiently while SEM excels in hiding such features very efficiently. For instance, Fig. 7a showing the width of the deposited coffee-stain ring displayed in Fig. 1a whereas line scans of HIM and SEM images in the images shows contrasting behaviour for both techniques. The red curve (line scan of Fig. 1b) in Fig. $7 \mathrm{~b}$ shows no deposits of nanoparticles nearly up to $2.5 \mu \mathrm{m}$ range whereas blue cure (line scan of Fig. 1c) in the similar figure shows such deposits right from the beginning of the coffee-stain ring. This also confirms that HIM sees more of CTAB than the SEM.

It has been observed that everywhere within the coffee-stain ring that gold nanorods are self-assembled in side-by-side ordered arrays. Such arrays are very much predictable in suspension owing to the stronger Van der Waals attractive forces between the two metal nanorods [19]. HIM and SEM images in Figs. 1 and 2 for that reason showed nicely assembled side-by-side gold nanorods. Close examination of these images clearly suggests that HIM shows more gluing kind of side-by-side arrays whereas SEM images display neatly arranged nanorods. In the relatively cleaner suspensions HIM can image gold nanorods just like SEM but still contrasting side-by-side arrays can be seen in. Such ordered arrays are very much different from the images displayed in Fig. 3. To evaluate further this analogy, line scans for side-by-side placed nanorods are presented in Fig. 8a, b. line scan in Fig. 8a (red curve) showing a lot of noise within the these side-by-side oriented gold nanorods whereas Fig. $8 \mathrm{~b}$ (blue curve) plotted using SEM image showing nearly no noise. Indicating as if there is nothing in between two nanorods placed side-by-side in SEM image whereas HIM indicating existence of something within the similar arrangements.

\section{Conclusions}

In this work, various imaging techniques such as SEM, HIM, AFM, and STM are performed for the study of CTAB coated gold nanorod deposits. Contrasting images of HIM and $\mathrm{SEM}$ on $\mathrm{SiO}_{2}$ substrate can be summarized in couple of points:

i. For large CTAB molecules in suspension, SEM generally excels in imagining the gold nanorods whereas HIM could not able to highlight the gold core everywhere within the deposits efficiently.

ii. For relatively low CTAB contents in the supernatant with nanorods, HIM can image gold nanorods but then again display some kind of gluing material between the nanorods which is not observed in the SEM images.

AFM and STM analysis of nanoentities on the HOPG surface shows that CTAB molecules deposit everywhere on the substrate. It has been observed that in some places on the HOPG terraces gold nanorods were observed buried under the large layers of CTAB whereas at some other locations deposited nanorods were found visible under the thin self-assembled layers of CTAB.

This study will be helpful to highlight the true picture of self-assembled arrays of CTAB stabilized gold nanorods and consequently will help to understand the possible blockade offered by the excess CTAB molecules that are normally hidden in SEM images. Moreover, AFM shows CTAB coverages between gold nanorods and the substrate will effect surface-particle interaction whereas CTAB layers on top of nanorods will interfere the signal coming from the top. Therefore, true knowledge of CTAB presence all over the surface as well as around the gold nanorods will be helpful to materialize precise approximation of the relevant uses expected from the gold nanorod self-assembly. In that respect, CTAB stabilized gold nanostructures exploration merely by SEM is misleading and requires more investigation.

Acknowledgements Some of the images used in this work are taken from my PhD thesis entitled "Evaporation induced self-assembly and characterization of gold nanorods". The research is carried out under the faculty development program, University of Peshawar, Peshawar KP, Pakistan. 


\section{Compliance with ethical standards}

Conflict of interest The authors declare that they have no conflict of interest.

\section{References}

1. Knight MW, Sobhani H, Nordlander P, Halas NJ (2011) Photodetection with active optical antennas. Science 332:702

2. Yan B, Boriskina SV, Reinhard BM (2011) Design and implementation of noble metal nanoparticle cluster arrays for plasmon enhanced biosensing. J Phys Chem C 115:24437

3. Bagheri S, Weber K, Gissibl T, Weiss T, Neubrech F, Giessen H (2015) Fabrication of square-centimeter plasmonic nanoantenna arrays by femtosecond direct laser writing lithography: effects of collective excitations on SEIRA enhancement. ACS Photonics 2:779

4. Karg M, König TAF, Retsch M, Stelling C, Reichstein PM, Honold T, Thelakkat M, Fery A (2015) Colloidal self-assembly concepts for light management in photovoltaics. Mater Today 18:185

5. Chen T, Reinhard BM (2016) Assembling color on the nanoscale: multichromatic switchable pixels from plasmonic atoms and molecules. Adv Mater 28:3522

6. Pastoriza-Santos I, Kinnear C, Perez-Juste J, Mulvaney P, LizMarzan LM (2018) Plasmonic polymer nanocomposites. Nat Rev Mater 3:375

7. Kraus T, Brodoceanu D, Pazos-Perez N, Fery A (2013) Colloidal surface assemblies: nanotechnology meets bioinspiration. Adv Funct Mater 23:4529

8. Christoph H, Eric HH, David VL, Guillermo GR, Cristiano M, Agustín M, Luis MLM (2019) Solvent-assisted self-assembly of gold nanorods into hierarchically organized plasmonic mesostructures. ACS Appl Mater Interfaces 11:11763

9. Ahmad I, Jansen HP, Zandvliet HJW, Kooij ES (2016) Hydrodynamic confinement and capillary alignment of gold nanorods. Nanotechnology 27:025301

10. Ahmad I, Jansen HP, van Swigchem J, Ganser C, Teichert C, Zandvliet HJW, Kooij ES (2015) Evaporative gold nanorod assembly on chemically stripe-patterned gradient surfaces. J Colloid Interface Sci 449:261-269

11. Ahmad I, Derkink F, Bampoulis P, Boulogne T, Zandvliet HJW, Jan R, Khan H, Kooij ES (2017) Step-edge induced orientation of nanorods in evaporative self-assembly on HOPG. Colloid Interface Sci Commun 19:25-30

12. Leonov AP, Zheng J, Clogston JD, Stern ST, Patri AK, Wei A (2008) Detoxification of gold nanorods by treatment with polystyrenesulfonate. ACS Nano 2(12):2481-2488

13. Patel JP, Zhao CX, Deshmukh S, Zou GX, Wamuo O, Hsu SL, Schoch AB, Carleen SA, Matsumoto D (2016) An analysis of the role of reactive plasticizers in the crosslinking reactions of a rigid resin. Polymer 107:12-18

14. Patel JP, Xiang ZG, Hsu SL, Schoch AB, Carleen SA, Matsumoto D (2015) Path to achieving molecular dispersion in a dense reactive mixture. J Polym Sci Part B Polym Phys 53:1519-1526

15. Patel JP, Xiang ZG, Hsu SL, Schoch AB, Carleen SA, Matsumoto $D(2017)$ Characterization of the crosslinking reaction in high performance adhesives. Int J Adhes Adhes. 78:256-262

16. Da Silva EC, Da Silva MGA, Meneghetti SMP, Machado G, Alencar MARC, Hickmann JM, Meneghetti MR (2008) Synthesis of colloids based on gold nanoparticles dispersed in castor oil. J Nanoparticle Res 10(1):201-208
17. Patel JP, Deshmukh S, Zhao C, Wamuo O, Hsu SL, Schoch AB, Carleen SA, Matsumoto D (2017) An analysis of the role of nonreactive plasticizers in the crosslinking reactions of a rigid resin. J Polym Sci Part B Polym Phys. 55(2):206-213

18. Patel JP, Hsu SL (2018) Development of low field NMR technique for analyzing segmental mobility of crosslinked polymers. J Polym Sci Part B Polym Phys 56:639-642

19. Ahmad I, Zandvliet HJW, Kooij ES (2014) Shape-induced separation of nanospheres and aligned nanorods. Langmuir 30:7953-7961

20. Ahmad I, Derkink F, Boulogne T, Bampoulis P, Zandvliet $\mathrm{HJ}$, Khan HU, Jan R, Kooij SE (2019) Self-assembly and wetting properties of gold nanorod-CTAB molecules on HOPG. Beilstein J Nanotechnol. 10:705

21. Hong L, Cacciuto A, Luijten E, Granick S (2006) Clusters of charged Janus spheres. Nano Lett 6:2510

22. Clark D, Tien J, Duffy DC, Paul KE, Whitesides GM (2001) Selfassembly of $10-\mu \mathrm{m}$-sized objects into ordered three-dimensional arrays. J Am Chem Soc 123:7677

23. Ahmad I, Jan R, Khan HU, Khan SA (2019) Nano gold shapeseparation effect induced by the motion of a droplet. Surf Interfaces 17:100342

24. Ahmad I, Khan HU, Jan R, Khan SA (2019) Anisotropic gold nanoparticles crystalizes in multifaceted superstructures. Mater Res Express 6:105006

25. Ahmad I, Jan R (2019) Size-separation and self-assembly of anisotropic nanoparticles in a coffee-stain ring. NANO 14(12):1950149

26. Karakoti AS, Das S, Thevuthasan S, Seal S (2011) PEGylated inorganic nanoparticles. Angew Chem Int Ed 50:1980-1994

27. Sperling YRA, Parak WJ (2010) Surface modification, functionalization and bioconjugation of colloidal inorganic nanoparticles. Philos Trans R Soc A 368:1333-1383

28. Cao J, Galbraith EK, Sun T, Grattan KTV (2012) Effective surface modification of gold nanorods for localized surface plasmon resonance-based biosensors. Sens Actuators B 169:360-367

29. Xu X, Heinig KH, Möller W, Engelmann HJ, Klingner N, Gharbi A, Tiron R, von Borany J, Hlawacek G (2019) Morphology modification of Si nanopillars under ion irradiation at elevated temperatures: plastic deformation and controlled thinning to 10 nm. Semicond Sci Technol 35(1):015021

30. Ghaderzadeh S, Ghorbani-AsI M, Kretschmer S, Hlawacek G, Krasheninnikov AV (2020) Channeling effects in gold nanoclusters under He ion irradiation: insights from molecular dynamics simulations. Nanotechnology 31:035302

31. Mousley M, Eswara S, De Castro O, Bouton O, Klingner N, Koch CT, Hlawacek G, Wirtz T (2019) Stationary beam full-field transmission helium ion microscopy using sub-50 keV He+: projected images and intensity patterns. Beilstein J Nanotechnol 10:1648-1657

32. Hlawacek G, Ahmad I, Smithers MA, Kooij ES (2013) To see or not to see: imaging surfactant coated nano-particles using HIM and SEM. Ultramicroscopy 135:89-94

33. Ahmed W, Kooij ES, van Silfhout A, Poelsema B (2010) Controlling the morphology of multi-branched gold nanoparticles. Nanotechnology 21(12):125605

34. Lohse SE, Murphy CJ (2013) The quest for shape control: a history of gold nanorod synthesis. Chem Mater 25(8):1250-1261

35. Ye X, Gao Y, Chen J, Reifsnyder DC, Zheng C, Murray CB (2013) Seeded growth of monodisperse gold nanorods using bromide-free surfactant mixtures. Nano Lett 13(5):2163-2171

36. Grzelczak M, Pérez-Juste J, Mulvaney P, Liz-Marzán LM (2008) Shape control Gold nanoparticle synthesis. Chem Soc Rev 37(9):1783-1791 
37. Ahmed W, Laarman RPB, Hellenthal C, Kooij ES, van Silfhout A, Poelsema B (2010) Dipole directed ring assembly of Ni-coated Au nanorods. Chem Commun 46(36):6711-6713

38. Bishop KJM, Wilmer CE, Soh S, Grzybowski BA (2009) Nano scale forces and their uses in self-assembly. Small 5(14):1600-1630

39. Dreaden EC, Alkilany AM, Huang X, Murphy CJ, El-Sayed MA (2012) The golden age: gold nanoparticles for biomedicine. Chem Soc Rev 41(7):2740-2779

40. Halas NJ (2010) Plasmonics: an emerging field fostered by nano letters. Nano Lett 10(10):3816-3822. https://doi.org/10.1021/ nl1032342

41. Chanana M, Liz-Marzán LM (2012) Coating matters: the influence of coating materials on the optical properties of gold nanoparticles. Nanophotonics 1(3-4):199-220. https://doi.org/10.1515/ nanoph-2012-0008

42. Hanrath $\mathrm{T}$ (2012) Colloidal nanocrystal quantum dot assemblies as artificial solids. J Vac Sci Technol A 30:030802

43. Tang Y, Chen S, Chen T, Guo W, Li Y, Mu S, Yu S, Zhao Y, Wend F, Gao F (2017) Synthesis of peanut-like hierarchical manganese carbonate microcrystals via magnetically driven self-assembly for high performance asymmetric supercapacitors. J Mater Chem A 5:3923

44. Huang X, Neretina S, El-Sayed MA (2009) Gold nanorods: from synthesis and properties to biological and biomedical applications. Adv Mater 21:4880

45. Li F, Lu J, Kong X, Hyeon T, Ling D (2017) Dynamic nanoparticle assemblies for biomedical applications. Adv Mater 29(14):1605897

46. Daniel M-C, Astruc D (2004) Gold nanoparticles: assembly, supramolecular chemistry, quantum-size-related properties, and applications toward biology, catalysis, and nanotechnology. Chem Rev 104:293

47. Liu BJ-W, Zheng J, Wang J-L, Xu J, Li H-H, Yu S-H (2013) Ultrathin W18049 nanowire assemblies for electrochromic devices. Nano Lett 13:3589

48. Kapur A, Aldeek F, Ji X, Safi M, Wang W, Cid AD, Steinbock O, Mattoussi H (2017) Self-assembled gold nanoparticle-fluorescent protein conjugates as platforms for sensing thiolate compounds via modulation of energy transfer quenching. Bioconjugate Chem 28:678

49. Vigderman L, Khanal BP, Zubarev ER (2012) Functional gold nanorods: synthesis, self-assembly, and sensing applications. Adv Mater 24:4811

50. Nobile C, Fonoberov VA, Kudera S, Torre AD, Ruffino A, Chilla G, Kipp T, Heitmann D, Manna L, Cingolani R, Balandin AA, Krahne $R$ (2007) Confined optical phonon modes in aligned nanorod arrays detected by resonant inelastic light scattering. Nano Lett 7:476

51. Bingi J, Nair RV, Vijayan C (2017) Time dependent Bloch mode transmittance in self-assembled random photonic crystal for photonic time delay switching. Opt Mater 64:95

52. Chen G, Wang D, Hong W, Sun L, Zhu Y, Chen X (2017) Fluorescence enhancement on large area self-assembled plasmonic-3D photonic crystals. Small 13:1602612
53. Vennerberg D, Lin Z (2011) Upconversion nanocrystals: synthesis, properties, assembly and applications. Sci Adv Mater 3:26

54. Yang N, You T-T, Liang X, Zhang C-M, Jiang L, Yin P-G (2017) An ultrasensitive near-infrared satellite SERS sensor: DNA selfassembled gold nanorod/nanospheres structure. RSC Adv 7:9321

55. Sreeprasad TS, Pradeep T (2011) Reversible assembly and disassembly of gold nanorods induced by EDTA and its application in SERS tuning. Langmuir 27:3381

56. Nizameev IR, Kadirov MK, Semyonov VA, Zakharova LY, Ismaev TI, Safiullin RA, Rizvanov IK, Babaev VM (2016) Palladium 1D nanoscale aggregates on a graphite surface using CTAB hemicylindrical micelle templates. Dalton Trans 45(27):11035-11041

57. Chun J, Li JL, Car R, Aksay IA, Saville DA (2006) Anisotropic adsorption of molecular assemblies on crystalline surfaces. $J$ Phys Chem 110:16624

58. Paruchuri VK, Nguyen AV, Miller JD (2004) Zeta-potentials of self-assembled surface micelles of ionic surfactants adsorbed at hydrophobic graphite surfaces. Colloid Surface Physicochem Eng Aspect 250:519

59. Shin $Y J$, Wang $Y$, Huang $H$, Kalon $G$, Wee ATS, Shen Z, Bhatia CS, Yang $H$ (2010) Surface-energy engineering of graphene. Langmuir 26:3798

60. Goyal PS, Aswal VK (2001) Micellar structure and inter-micelle interactions in micellar solutions: results of small angle neutron scattering studies. Curr Sci 80:972

61. Yang D-S, Zewail AH (2009) Ordered water structure at hydrophobic graphite interfaces observed by 4D, ultrafast electron crystallography. Proc Natl Acad Sci U S A 106(11):4122-4126

62. Imae T, Kamiya R, Ikeda S (1985) Formation of spherical and rodlike micelles of cetyltrimethylammonium bromide in aqueous $\mathrm{NaBr}$ solutions. J Colloid Interface Sci 108:215

63. Hashidzume A, Harada A, Kobayashi S, Müllen K (eds) (2015) Micelles and vesicles; Encyclopedia of polymeric nanomaterials. Springer, Berlin, Heidelberg, pp 1238-1241

64. Nikoobakht B, El-Sayed MA (2003) Preparation and growth mechanism of gold nanorods (NRs) using seed-mediated growth method. Chem Mater 15(10):1957-1962

65. Murphy CJ, Sau TK, Gole AM, Orendorff CJ, Gao J, Gou L, Hunyadi SE, Li T (2005) Anisotropic metal nanoparticles: synthesis, assembly, and optical applications. J Phys Chem B 109:13857-13870

66. Kadirov MK, Nizameev IR, Zakharova LY (2012) Platinum nanoscale lattice on a graphite surface using cetyltrimethylammonium bromide hemi- and precylindrical micelle templates. $J$ Phys Chem C 116:11326-11335

Publisher's Note Springer Nature remains neutral with regard to jurisdictional claims in published maps and institutional affiliations. 